\title{
The Role of CAD in Enterprise Integration Process
}

\author{
M. Ota, I. Jelínek
}

This article deals with the problem of the mutual influence between software systems used in enterprise environment and enterprise integration processes. The position of CAD data and CAx systems in the integrated environment of manufacturing enterprises is clarified. As a consequence, the key role of CAx systems used in those companies is emphasized. It is noted that the integration of CAD data is nowadays only on a secondary level, via primarily integrated PDM systems. This limitation is a reason why we are developing a unified communication model focused on product-oriented data. Our approach is based on Internet technologies, so we believe that is independent enough. The proposed system of communication is based on a simple request-replay dialogue. The structure of this model is open and extensible, but we assume supervision supported by an Internet portal.

Keywords: CAD, Enterprise Information Systems, Internet Portals, PDM.

\section{Introduction}

In recent times we have been witnessing to a strong trend toward enterprise integration. This tendency is a result of the increasing efficiency of enterprises, which is a logical consequence of classic competition among companies. Enterprise integration processes are related to the integration of enterprise software systems [1]. Modern information technologies are being used increasingly by businesses. During the 1990 s the situation changed with the introduction of internet-oriented technologies not only in the academic sphere, but also in commerce. This change meant that each enterprise has started to be physically joined by this computer network. Users of enterprise software and, in particular, managers have started to call for a logical link-up of territorially isolated software equipment. This has speeded up mutual communication among software systems and has led to integration. At the same time, the globalisation of world industry and the international economy initiated massive enterprise integration processes and intensive enterprise collaboration. The new methods of collaboration (both internal and external) are solidly based on communicating software systems [2]. Thus in this period many standards, communication mechanisms and protocols have been developed, which effectively support software communication and formal integration [3].

The typical schema of the architecture of enterprise software systems is that the contemporary model is based on a complex Enterprise Information System (EIS), which communicates with other software. EIS logically wraps all the enterprise software and this shell is integrated on the information level. In present day applications of Internet mechanism, EIS is not applied only to the communication layer, but also to the presentation layer. Solutions based on this new approach are usually called portal solutions or Internet portals. The phenomenon of portal solutions also enables more heterogeneity of hardware platforms [1]. This is very important, because the spectrum of EIS users is widely extended, and territorial boundaries of EIS are more suppressed than in the past. Typical example is the field-worker who is using a mobile device (such as a mobile telephone) connected to EIS via Internet.

The portal solutions used in an enterprise environment, can be classified into two basic groups. The first is an Enterprise Application Portal (EAP) while the second is an Enterprise Information Portal (EIP) [4]. EIP's aim is to provide information (and are more widespread - see Fig. 1), EAP are

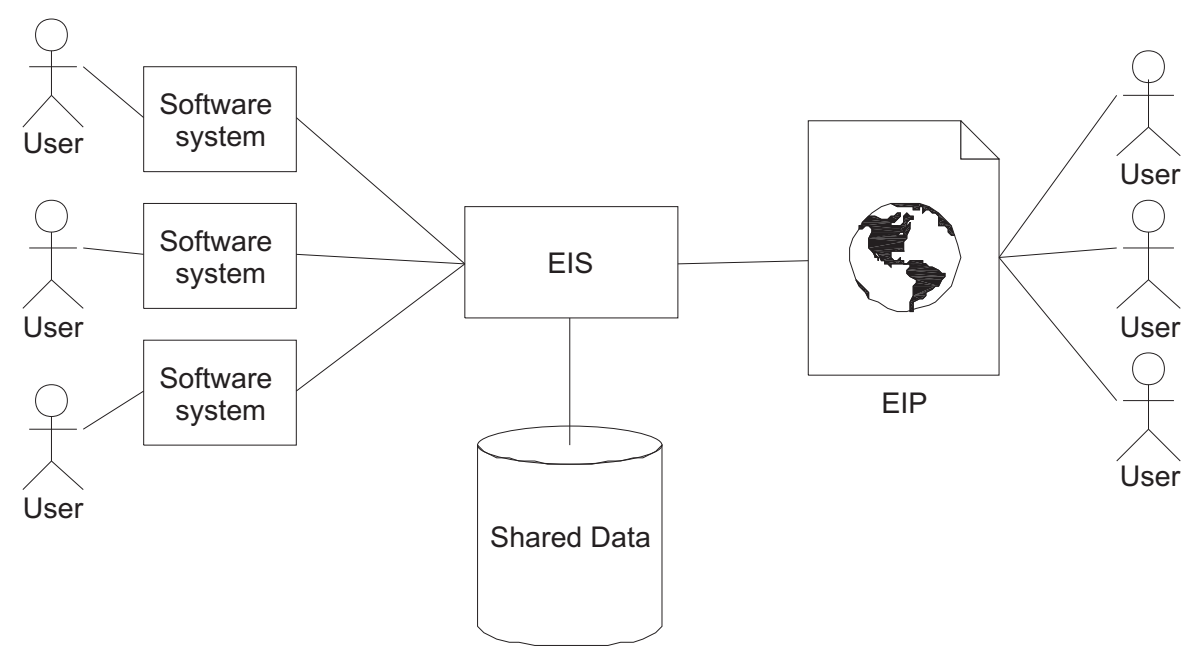

Fig. 1: Enterprise information portal 


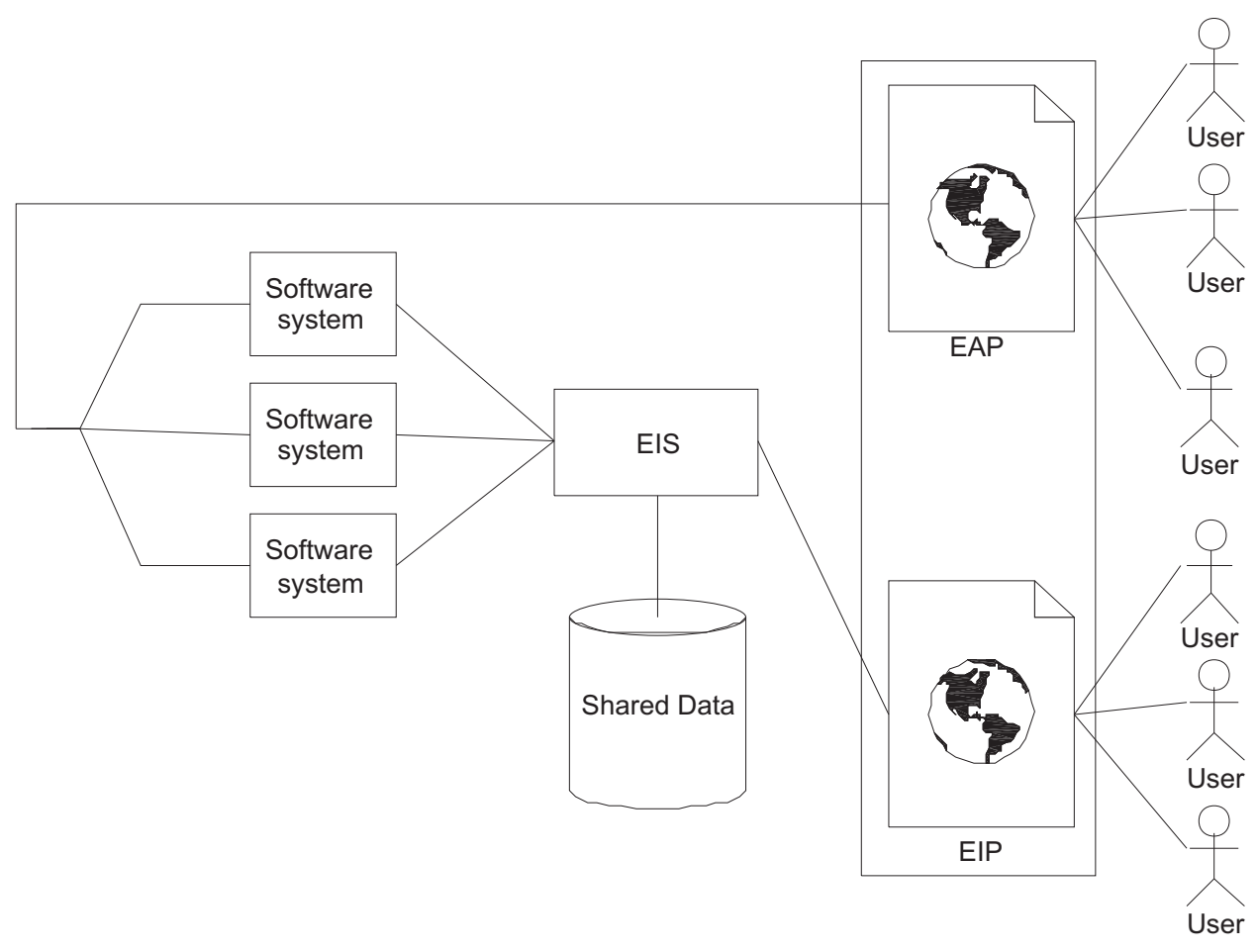

Fig. 2: Enterprise application portal

gateways for application runs (and are usually combined with an EIP one - see Fig. 2). However this classification is disputable, because EIPs are also built on some application logic. The use of enterprise software applications via an Internet portal is under intense study in the fiels known as Enterprise Application Integration (EAI).

Each manufacturing enterprise stores, manages and uses the data about the products that it produces. This data is called product-oriented data. The data special parts created by CAD systems are product oriented data. These data is specific, so the software systems that operate on this data (known generally as CAx systems, especially CAD, CAM and CAE) must be perceived as special in the context of data sharing and information flow. These software systems play a major role in all technically oriented companies. CAx software is intensive on computer power, so the integration to the EAP does not yet have good results. However, content integration to the context of the information system is desirable. There are ways to solve this, but we believe that the degree of integration is not sufficient and we suppose there are other ways that could markedly improve the integration of CAx systems. The products of a manufacturing company are at the centre of its concern and all the CAx data is just about the product. Integration of the CAx technologies, vitalized by the integration of enterprise processes, improves the support for teamwork and makes necessary information about the products available to CAx users, such as managers, marketing workers, etc. CAx systems must therefore be designed as open systems, in order to be able to integrate them into the enterprise information system.

Last but not least, there is the problem of using more than one CAx system on the same areas (e.g. two or more CAD systems). No CAx system is generally best, and many companies use a variety of CAD systems for different parts of the design process e. g. for sheet metals and metal casting support. As a result of this specialisation there are heterogeneous subsets of product data for a single product, and there is no easy way to exchange related information between two different CAD systems.

\section{Observation}

Interoperability and data connectivity of enterprise software equipment is an area where dynamic developments are taking place. There are complex methodologies for data-oriented and data-intensive software integration, such as agendas, databases and data warehouses. However, CAx systems are excluded from the focus of primary software integration processes. Since computer-aided design is widely used in the enterprise environment of manufacturing companies, the gap between the CAD systems, on the one hand, and the rest of the company and its other software equipment, on the other hand is a serious problem. Relationships between CAD and other CAx software have been solved more or less satisfactory (partly out outside the wrap of EIS).

The field of Product Data Management (PDM) tries to systematize and manage data about products, including CAx data [5]. PDM systems manage the storrage of product data, both attribute, and documentary product data, as well as relationships between them. This is usually implemented through a relational database system. Data classification should be a fundamental capability of PDM systems. CAD data is therefore secondarily integrated into EIS via a primarily integrated PDM system. This type of integration is less intensive, not so close, and the efficiency of integration of CAD data soon become weak. The binding among CAD data and EIS is not always complete and fully up to date. Although all production data including CAD data, is stored in PDM (e.g., files with virtual 3D models), much information is not reachable (e.g., some characteristics and/or physical relations 
among parts of virtual 3D models). A typical situation that illustrates the problem of managing this data is when a manager or a salesman needs to know some physical characteristic of a manufactured product. He is able to find the appropriate CAD file via EIS - thanks to collaboration with the integrated PDM. Even the desired characteristic is easy to obtain, but only in the CAD system in which the model was created. However managers are not able to use CAD systems (and why should they have such skills?). They therefore rely on some other employee - a CAD-worker. Although touch PDM systems may try to store as much information as possible (including data extracted from CAD model), it is not feasible to cover every potential user query. Moreover, there is spread data redundancy in the present model, which implies many known problems. We are convinced that a better compromise between complexity and efficiency of retrieving information held in CAD data can be found.

\section{Proposal}

The main aim of our research is to improve the integration of product data into an enterprise information environment managed by EIS. We would like to move the integration of CAx data from the secondary level of integration in EIS to the primary level. This does not mean any radical changes, such as eliminating of PDM systems, but involves making information contained by CAD data available. This information can be released by the possibility of communicating between the CAD software, that is appropriate to a particular data record (with an operational relation to this record) and other software, that needs obtain this information and/or mediates it to the user.

The model of an enterprise environment that we propose integrates CAx systems more tightly into EIS. The organisation and the architecture of EIS and their integration with other software remain the same. The PDM system is also used in accordance with the former model, and even specific PDM software can be retained. PDM system is used for classifying and managing of data storage, but the set of redundant information obtained from CAx data stored in PDM is minimized in our model (it is reasonable to store only frequently queried information). Other product-oriented queries are solved in collaboration with an appropriate CAx system. Obviously, there is a need for a new mechanism for querying information contained in CAx data, which is the only way in which our differs from the present day model.

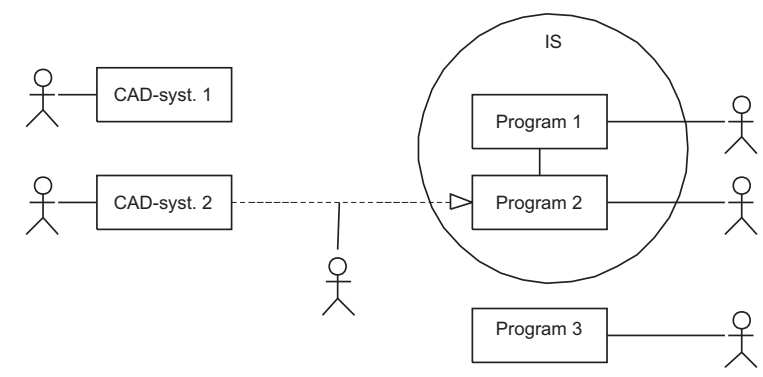

Note: This shows communication between two systems only. There should be similar communication among all programs able to communicate with CAD.

Fig. 3: Currently used model

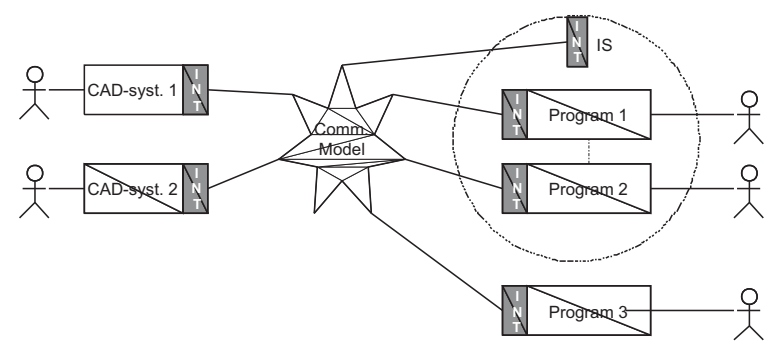

Fig. 4: Proposed model

The compared current state and the proposed state of communication between CAD systems and enterprise software is shown in Figs. 3 and 4. Communication between two different CAD systems is the same scenario as communication between a CAD system and other software - there is usually more than one CAD system in use in a real enterprise. There is only a higher probability that the CAD systems could share part of the spectrum of supported export/import file formats.

Fig. 3 shows a present-day approach. Communication is shown between CAD system and another program. The need for user assistance is quite common, so it is indicated in the figure. If the figure were to describe full communication, there would be a full graph, and each program would have to implement communication mechanisms for each CAD system, and for each other program. This heterogeneity is a reason for the present-day use of lower complexity of automatic connectivity. Thus there are various types data or information flow channel in present-day communication models, sometimes even a human being (for this reason there is a dashed line in the figure). The circle shows the imaginary boundary of software integration to EIS (systems wrapped by EIS are connected by solid line, because first-level integration is applied at this point).

Fig. 4 is illustrates the proposed model. Each program that would communicate with some CAD system (i.e., obtaining information from CAD data) should be extended by an interface for using the communication model. This extension can customised - not only by a CAD system producer, but also by the individual, who implements the complex software solution of an enterprise. CAD systems must therefore be open/extensible as noted above. There is only one interface for all product data oriented communication among the entire participating software. If some program does not implement the interface of the communication mechanism, and retains the architecture shown in Fig. 3, this software will not be excluded from communication, because it will still be allowed to use the former mechanism. This is significant, because it means that movement from the present model to the proposed model can be performed stepwise. Fig. 4 describes communication among all shown programs and CAD systems, unlike the structure presented in Fig. 3 - the graph of the software connection is much simple due to the unified communication channel. The circle showing the level of integration boundary is dashed, signifying that this boundary makes no relevant sense.

The text above indicates that the described problem with communication is especially related to CAD. However if a CAx system implements the interface of the proposed model, it can fully use the new communication approach, and can provide data for it. 


\section{Model of communication}

The proposed model of communication relies on the ubiquity of the Internet in the business environment. It is a simple communication protocol based on TCP/IP protocols, such as HTTP. Only a clear structure and the scenario of interaction are defined. A real system of communication is based on a system of requests and replies. Requests are product-oriented queries. There are two basic types of queries:

- Property (e.g., the weight of a part, or is the IGES conversion format supported),

- List of available items (e.g., get supported conversion formats).

The categories of queries can be classified into several classes, such as physics (e.g., get weight or get material info), presentation (e.g., get VRML presentation), conversion (e.g., convert to STEP or get supported conversion formats), and others. Replies can be classified into three basic types:

- Result (e.g., $12.5 \mathrm{~kg}$, or VRML-file),

- Exception (e.g., 2D model without weight info),

- Error (e.g., appropriate CAD system for query solving not found).

The level of independence of this communication model is high - the only requirement is for the existence of TCP/IP protocols. There is no component technology, such as the need for CORBA, DCOM, J2EE or NET, which is problem in some enterprise communication systems $[6,7]$. Our model can be used in very heterogeneous environment - at both software and/or hardware levels.

A disadvantage of this communication model is the need to have appropriate CAD software installed in at least one computer connected to the EIS, because this software is needed for solving queries. However if the PDM system, in the present-day approach, stores some CAD files, software must also be installed, because otherwise the stored data is not useful. Thus this disadvantage is debatable.

It is evident that the draft of the communication protocol can neither encompass each field of the CAD application sphere, nor fully cover each field. Thus the model has to remain an open and extensible system. This fact prevents the model beeing definitely incomplete and to makes it applicable on any evolution in computer aided design and/or sphere of EIS. However, every open and/or extensible system is problematic, because its evolution tends to be unsystematic and parallel. The system is often not useful, because each user demands incompatible extensions of the model. For the reason we intend the supervise extension to the system. The first precaution considers the definition of exact rules for extending the model, as these rules are the (extensible) part of system. The second basic element of supervision is designed in the form of the Internet portal of the communication model (EIP type - see Fig. 1). This portal solution allows users to make a simple and unambiguous extension automatically or semi-automatically. It will also be able to collect requests, suggestions, comments, etc., from users. These inputs will be evaluated and processed and the results will be released by the portal (perhaps in the form of an extension of the model). Compatibility among users will be ensured by a system of questions (e.g., is the IGES conversion format supported) or by results in the form of exceptions (e. g., get weight $\rightarrow 2 D$ model without weight info). A versioning system will also be helpful.

\section{State of our research and future work}

We have completed the basic design of the communication model, as described above. A part of our model is a draft of its lifecycle supported by an Internet portal. We will to keep this model extensible and open, but with Internet-powered supervision. The system of supervision is not being tested. This experimental phase is based on Microsoft NET technology, but the system of support is planned to be independent from this platform for users, so it should be no problem to change this background of the lifecycle of our model. We are considering using the Web Services technology [8] as an addition to the classic portal solution.

The first problem, that has still not been full satisfactorily solved is that we have to harmonize our proposed model with existing standards, such as STEP - ISO 10303 [9]. Our model cannot ignore world standardisation processes, but it should remain simple, effective and usable.

The second problem concerns efficient use of computer resources. If each CAD remains in operation, communication will not be problematic. However, the software is invoked and is stopped, there will be a waste of computer power during cyclic starting and exiting from the systems (Fig. 5). In the case of two (or more) queries applied on a single CAD model, the problem will be considerable. If the communication model is to be effective in query-intensive applications, this problem will have to be solved.

We will now start experiments on the proposed model. In first trial phase we plan to use Autodesk CAD systems and .NET technologies (APS.NET, C\# and VB(A)) to simulate enterprise information system environment. Other CAD systems and other EIS platforms (such as Java and J2EE) will be included in experiments in subsequent phases of our laboratory experiment.

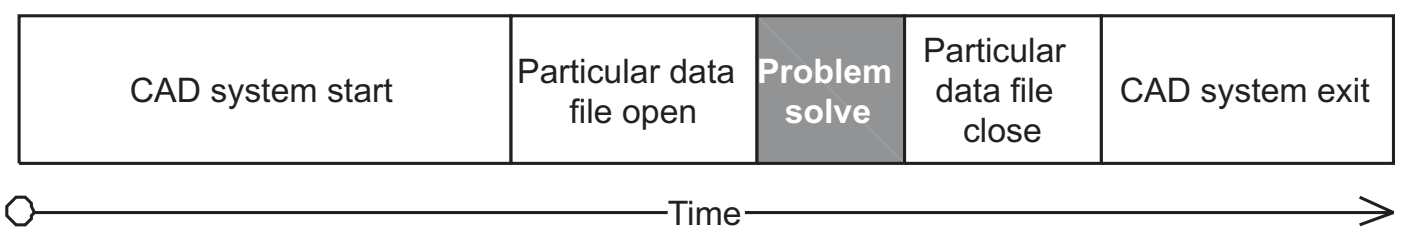

Fig. 5: Usual plan for solving an information query to the CAD data file 


\section{Conclusion}

Present-day methods for integrating of CAx software are very limited, because there is a great deal of unreachable information in CAD data (for non CAD-system users).

Our research focuses on the problem of communication among enterprise software, between CAD systems and other programs. As a result we are developing a unified communication model focusing on the product data of manufacturing enterprises. We have the formal draft of this model; and we are harmonizing it with existing standards used in the product data oriented sphere. We are aware of the computer power intensity problem, and are now working on solving it.

\section{Acknowledgment}

This research has been supported by GAČR grant No. 102/01/0723.

\section{References}

[1] Ota M.: The Influence of Enterprise Integration Processes on Software Systems. Acta Polytechnica. Prague: accepted.

[2] Ota M., Jelínek I.: Simulated Enterprise Information System (SEIS). Proceedings of Workshop 2003. Prague: CTU, 2003, Vol. A, p. 108.

[3] Ota M.: What Is Covered in the Core of Information Systems. (In Czech). It-System. CCB. Brno: accepted, in print 2003, No. 6.
[4] Řepa V.: Business Processes and Evolution of Information Systems. In Czech. Systémová integrace. ČSSI. Prague: 2002, No. 2, p. 7.

[5] PDM Information Centre: http://www.pdmic.com/ as April 2003.

[6] Michalčík L., Jelínek I.: Advanced Technologies and Standards in the Area of Integration CAD and IS. Proceedings of Workshop 1999. Prague: CTU, 1999, p. 89.

[7] Bohms M., Van Der Waal J.: Combining STEP, JAVA and Mapping gives user control of data. EPM Technology, The EXPRESS way. No. 2, 1998.

[8] Kačmář D.: We Are Programming the .NET Applications in Visual Studio .NET. In Czech. Computer Press, Praha. 2001.

[9] Al-Timini K., Mackrell J.: STEP - Towards Open Systems. STEP Fundamentals \& Business Benefits. CIM data, 1986.

\section{Martin Ota}

fax: +420 224923325

e-mail: otam@fel.cvut.cz

Prof. Dr. Ivan Jelínek

Dept. of Computer Science and Engineering

Czech Technical University

Faculty of Electric Engineering

Karlovo nám. 13

12135 Praha 2, Czech Republic 